\title{
The Effects of Applying Apparent Diffusion Coefficient Parameters on the Differentiation between Fourth Ventricular Ependymoma and Diffuse Intrinsic Pontine Glioma
}

\author{
Nguyen Minh Duc ${ }^{1,2,30}$ \\ ${ }^{1}$ Department of Radiology, Hanoi Medical University, Hanoi, Vietnam \\ 2 Department of Radiology, Pham Ngoc Thach University of Medicine, \\ Ho Chi Minh City, Vietnam \\ ${ }^{3}$ Department of Radiology, Children's Hospital 02, Ho Chi Minh City, \\ Vietnam \\ J Child Sci 2020;10:e169-e174.
}

\begin{abstract}
Address for correspondence Nguyen Minh Duc, MD, Department of Radiology, Pham Ngoc Thach University of Medicine, Ho Chi Minh City, Vietnam (e-mail: bsnguyenminhduc@pnt.edu.vn).
\end{abstract}

Distinguishing the fourth ventricular ependymoma from diffuse intrinsic pontine glioma (DIPG) is essential to improve the treatment strategy between these two tumor types. We attempted to evaluate the effects of applying apparent diffusion coefficient (ADC) values to the distinction between pediatric fourth ventricular ependymomas and DIPGs. Brain magnetic resonance imaging, including diffusion-weighted imaging and $A D C$, was assessed in 26 patients, who were divided into two groups: group 1 included 8 patients with fourth ventricular ependymoma and group 2 included 18 patients with DIPG. The MannWhitney $U$ test was utilized to compare tumoral maximum ( $A D C m a x)$, minimum ADC ( $A D C$ min), mean $A D C$ (ADCmean), and standard deviation (ADCsd) values, and the ratios between the tumor and parenchyma values for each of these parameters (rADCmax, rADCmin, rADCmean, and rADCsd, respectively) between the two groups. Cutoff values were calculated based on receiver operating characteristic curve analysis and the Youden index, and the area under the curve (AUC), sensitivity, and specificity were determined. The median $A D C$ max, $A D C$ min, $A D C$ mean, $r A D C$ max, $r A D C$ min, and $r A D C$ mean values were significantly lower in group 1 than in group $2(p<0.05)$. For the differential diagnosis of ependymomas and DIPGs, a cutoff ADCmean value of $1.02 \times 10^{-3} \mathrm{~mm}^{2} / \mathrm{s}$ was determined, which produced a sensitivity of $100 \%$, a specificity of $88.9 \%$, and an AUC of $95.8 \%$. ADC parameters should be considered when performing a differential diagnosis between fourth ventricular ependymomas and DIPGs. Based on our findings, a cutoff ADCmean value of $1.02 \times 10^{-3} \mathrm{~mm}^{2} / \mathrm{s}$ was the most significant and effective parameter for this purpose.

\section{Introduction}

Central nervous system tumors in children, including supratentorial and infratentorial tumors, are the second-most prevalent types of cancer, after hematological malignancies. Tumors most commonly occur in the infratentorial area, with

received

July 25,2020

accepted after revision

September 20, 2020
DOI https://doi.org/

10.1055/s-0040-1718897. ISSN 2474-5871.
$60 \%$ of all pediatric brain tumors being found in this location. The most prevalent type of tumor in the brain stem is diffuse intrinsic pontine glioma (DIPG), whereas ependymoma is most commonly located in the fourth ventricle. ${ }^{1,2}$

Kasliwal and Agrawal noted that one patient had an ependymoma-consistent posterior fossa tumor. However, a
Copyright $\odot 2020$ Georg Thieme Verlag License terms KG Stuttgart · New York
(1) (1) 
mildly vascular intrinsic brainstem glioma was identified intraoperatively. Postoperatively, an astrocytoma diagnosis was verified by histopathology. ${ }^{3}$ Thakar et al reported a patient with a diffuse lesion, composed of intrinsic and symmetric exophytic cisternal components in the pontomesencephalic region consistent with diagnosis of DIPG. Nevertheless, the histopathological analysis revealed a confirmation of ependymoma. ${ }^{4}$ According to Jallo et al, although ependymomas are primarily extra-axial, they can mimic an intrinsic brain stem tumor, inducing compression on the brain stem or insinuating the Luschka foramen into the brain stem through lateral extension. Occasionally, ependymomas can arise as medullary lesions, resulting in the misdiagnosis of ependymoma as glioma of the brain stem. ${ }^{5}$ Furthermore, Tonn et al reported that $40 \%$ of ependymomas may present low-density necrotic centers, imitating malignant gliomas. ${ }^{6}$ The optimal therapeutic strategies and prognosis for ependymoma and DIPG differ; therefore, improving the distinction between ependymoma and DIPG may earn improved outcomes for patients. $1,2,5,6$

The gold standard diagnostic method for these tumor classifications remains pathological assessments following biopsy or surgical resection. However, even when using the gold standard method, sampling errors and interpretation variability can make diagnoses challenging. Additionally, biopsies are associated with a high risk of inducing morbidity and mortality. Preoperative imaging can be utilized to establish a diagnosis, a differential diagnosis, or to describe the tumor's anatomic location. ${ }^{1,2,7,8}$ Diagnosis based on preoperative imaging is the most popular, noninvasive process. Among various available imaging modalities, magnetic resonance imaging (MRI) is the most preferred method, owing to high intrinsic soft-tissue contrast, nonradioactive and noninvasive features, and the lack of any possible biological risks to patients. ${ }^{7,8}$ Strengthening associations between imaging-based diagnosis and tissuebased diagnosis can facilitate the expanded use of imaging methods for care planning. Markedly, earlier research examining the use of specific MRI sequences for the distinction among different types of growing posterior fossa tumors in children has shown encouraging results. ${ }^{7-13}$ Diffusion-weighted imaging (DWI) is a commonly utilized MRI sequence that plays a critical role in the differentiation of pediatric posterior fossa tumors, among the various available imaging sequences. ${ }^{7-13}$

DWI enables the calculation of the microscopic diffusion of water into tissues, resulting in the creating of apparent diffusion coefficient (ADC) maps, which represent the absolute measure of average diffusion in each voxel. ${ }^{7-13}$ Although previous studies have assessed the application of DWI to the differentiation of common posterior fossa tumors such as medulloblastoma, ependymoma, and pilocytic astrocytoma in children, knowledge about the utilization of DWI for the distinction between fourth ventricular ependymoma and DIPG was not abundant. ${ }^{9-13}$ Thus, in this clinical study, we attempted to assess the effects of utilizing ADC parameters for the differentiation between fourth ventricular ependymoma and DIPG.

\section{Materials and Methods}

\section{Subjects}

This prospective study was approved by the Institutional Review Board of Children's Hospital 02 (Ref: 352/NĐ2-CĐT). Informed consent was received from all patients' legal representatives before the MRI procedure. The study was performed at Children's Hospital 02, starting in February 2019, and lasted for 12 months. A total of 26 patients were enrolled in this study, divided into two groups (median age: 5 years; male/female: 17/9). Group 1 consisted of 8 fourth ventricular ependymoma patients, whereas group 2 included 18 DIPG patients. The median ages of groups 1 and 2 were 3.5 and 5.5 , respectively. The male/female ratios of groups 1 and 2 were $7 / 1$ and 10/8, respectively. Patients diagnosed with fourth ventricular ependymoma underwent surgery to obtain samples for histopathological verification. In terms of pros and cons of histopathological findings via surgery or biopsy for DIPG in children and as our hospital protocols, all patients with DIPG were diagnosed with DIPG based on a complete consensus between neuroradiologists and neurosurgeons. ${ }^{14-16}$

\section{MRI Procedure}

In this study, all patients were checked using a 1.5 Tesla MRI machine (Multiva, Philips, Best, the Netherlands) and judged utilizing the DWI sequence, with the following detailed parameters: repetition time (TR): shortest; echo time (TE): shortest; flip angle: 90 degrees; slice thickness: $5 \mathrm{~mm}$; gap: $1 \mathrm{~mm}$; field of view: $230 \times 230 \mathrm{~mm}$; matrix: $144 \times 90 \mathrm{~mm}$; plane: axial; number of acquisitions: 2 ; b values: $0,25,50,100,200,1,000$, and 1,500; duration: 3.43 minutes. ADC values were automatically derived from the DWI sequence, after finishing the scan.

\section{Variables}

A region of interest (ROI) was defined in both the tumor and the parenchyma on the ADC map, using the MR diffusion tool available in Philips Intellispace Portal, version 11. Clinician chose the slice where the tumor was max in size, later drew the ROI within the tumor covered solid part as large as possible (at least $100 \mathrm{~mm}^{2}$ ) and the ROI of the normal-appearing parenchyma at the same slice. All ADC values were calculated for both ROIs, including the maximum ADC (ADCmax), minimum ADC (ADCmin), mean ADC (ADCmean), and standard deviation ADC (ADCsd). The following additional parameters were also assessed: the ratios between the tumor and parenchyma values for each parameter were also calculated (rADCmax, rADCmin, rADCmean, and rADCsd) (-Figs. 1 and 2).

\section{Statistical Analysis}

Statistical analysis was performed using SPSS software version 26 (IBM Corp, Armonk, New York, United States). Continuous variables are presented as the median and interquartile range. The Mann-Whitney $U$ test was utilized for comparisons between quantitative variables. Receiver operating characteristic curve analysis and the Youden index were used to investigate the cutoff point, accuracy, sensitivity, and specificity. Differences with $p<0.05$ were considered significant. 


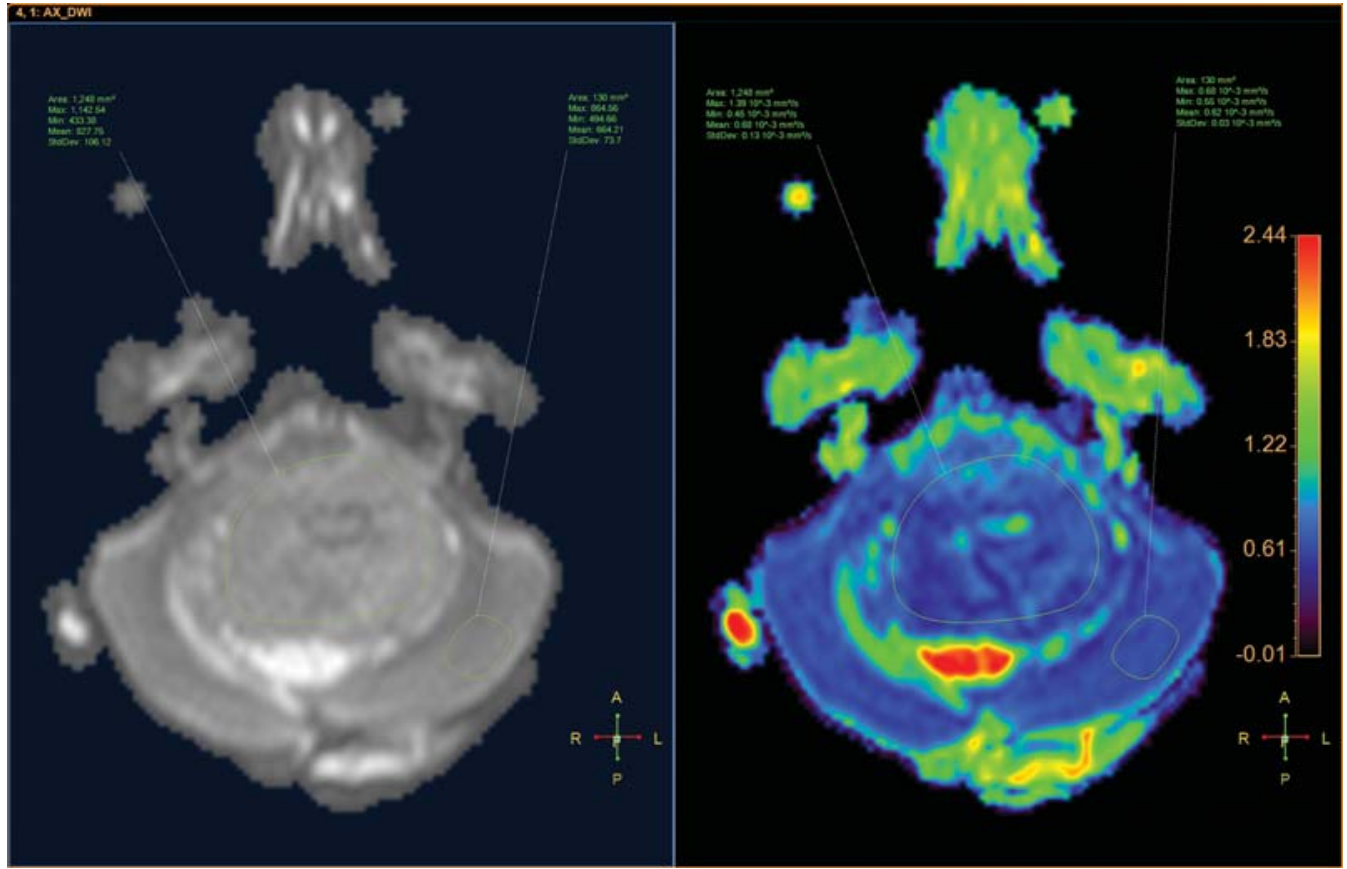

Fig. 1 A 4-year-old male patient, with a tumor inside the fourth ventricle, which was confirmed as ependymoma after surgery. (Left) Axial diffusion-weighted imaging. (Right) apparent diffusion coefficient map.

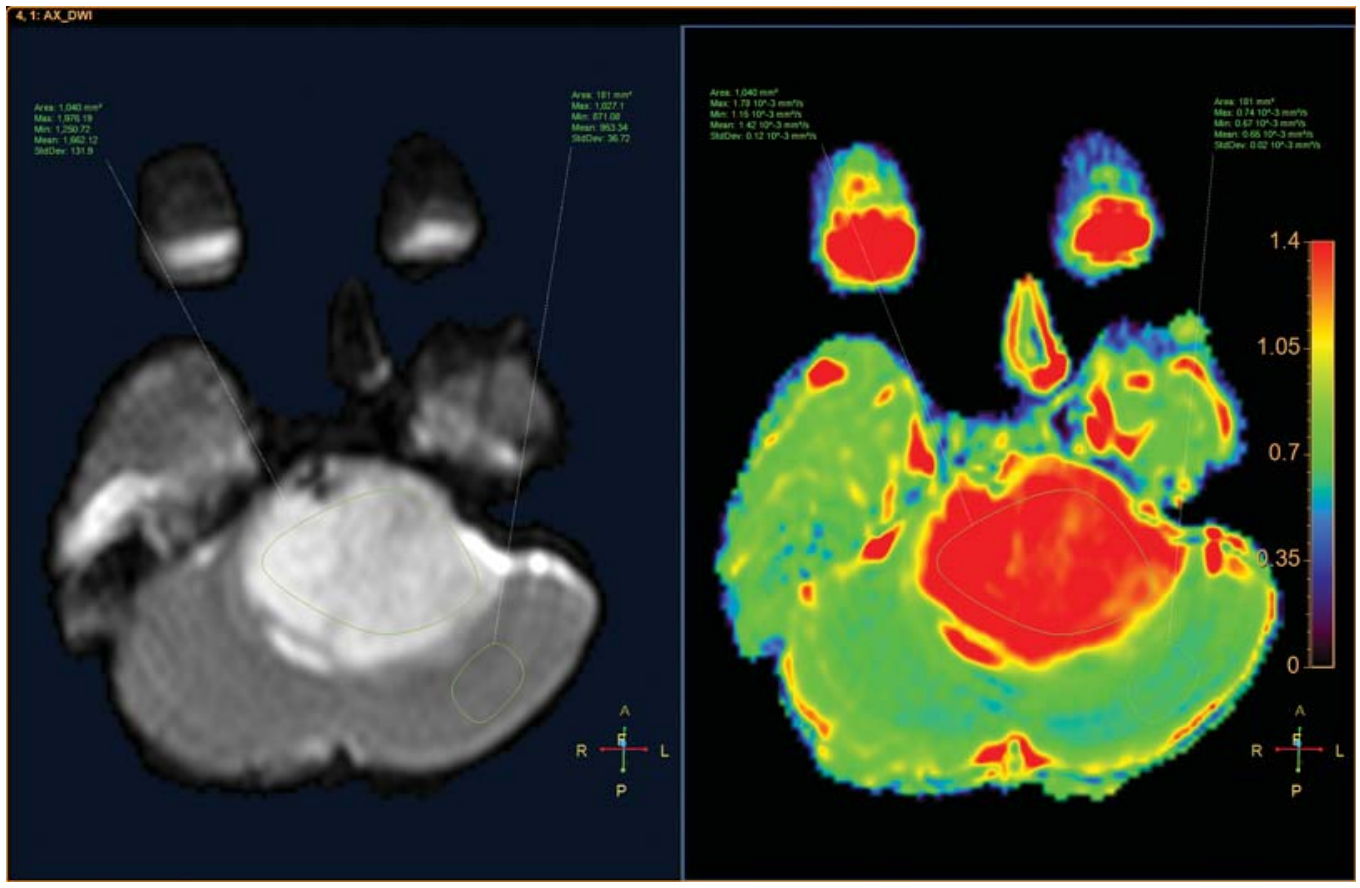

Fig. 2 A 6-year-old male patient, with a tumor located in the pons, which was diagnosed as a diffuse intrinsic pontine glioma. (Left) Axial diffusion-weighted imaging image. (Right) Apparent diffusion coefficient map.

\section{Results}

In group 1, there were four cases of classic ependymoma (grade II) and four cases of anaplastic ependymoma (grade III) meanwhile there was no histological grading in group 2. According to surgical reports, it is noted that all of ependymomas, in the present study, were originated from the floor of the fourth ventricle.

As shown in - Table 1, the ADCmax, ADCmin, ADCmean, rADCmax, rADCmin, and rADCmean values for fourth ventricular ependymomas were significantly lower than those for DIPGs $(p<0.05)$. 
e172 ADC Parameters Distinguish Fourth Ventricular Ependymoma from DIPG Minh Duc

Table 1 Comparison of the basic characteristics between fourth ventricular ependymomas and diffuse intrinsic pontine gliomas

\begin{tabular}{|l|l|l|l|}
\hline & $\begin{array}{l}\text { Fourth } \\
\text { ventricular } \\
\text { ependymoma } \\
(\boldsymbol{n}=8)\end{array}$ & $\begin{array}{l}\text { Diffuse intrinsic } \\
\text { pontine glioma } \\
(\boldsymbol{n}=18)\end{array}$ & $p$-Value \\
\hline ADC & $1.01(0.71)$ & $1.64(0.49)$ & $0.003^{\mathrm{a}}$ \\
\hline $\begin{array}{l}\mathrm{ADCmax} \\
\left(10^{-3} \mathrm{~mm}^{2} / \mathrm{s}\right)\end{array}$ & $0.62(0.42)$ & $1.05(0.34)$ & $0.001^{\mathrm{a}}$ \\
\hline $\begin{array}{l}\mathrm{ADCmin} \\
\left(10^{-3} \mathrm{~mm}^{2} / \mathrm{s}\right)\end{array}$ & $0.77(0.44)$ & $1.38(0.50)$ & $<0.001^{\mathrm{a}}$ \\
\hline $\begin{array}{l}\mathrm{ADCmean} \\
\left(10^{-3} \mathrm{~mm}^{2} / \mathrm{s}\right)\end{array}$ & $0.08(0.09)$ & $0.10(0.04)$ & 0.093 \\
\hline ADCsd & $1.48(0.80)$ & $1.98(0.54)$ & $0.017^{\mathrm{a}}$ \\
\hline ADC ratio & $1.13(0.77)$ & $1.81(0.67)$ & $0.002^{\mathrm{a}}$ \\
\hline rADCmax & $1.25(0.74)$ & $2.01(0.63)$ & $<0.001^{\mathrm{a}}$ \\
\hline rADCmin & $2.62(2.31)$ & $2.41(1.95)$ & 0.824 \\
\hline rADCmean & & & \\
\hline rADCsd & &
\end{tabular}

Abbreviations: $A D C$, apparent diffusion coefficient; maxADC, maximum $A D C$; minADC, minimum $A D C ; A D C$ ratio, the ratio between the tumor and parenchyma values of each ADC variable.

${ }^{a}$ Statistically significant.

As demonstrated in - Table 2, a cutoff ADCmean value of 1.02 was established for the differential diagnosis between fourth ventricular ependymomas and DIPGs, resulting in the highest sensitivity value of $100 \%$, a specificity of $88.9 \%$, and an area under the curve (AUC) of $95.8 \%$ (-Fig. 3 ).

\section{Discussion}

We evaluated the impact apply DWI for tumor differentiation because DWI facilitates the measurement of water molecules diffusion into and out of cells. The diffusion rate is governed by microstructural and tissue microdynamics. Tissue diffusion coefficients may be determined using ADC values. The passage of water across the cell wall is affected by the overall

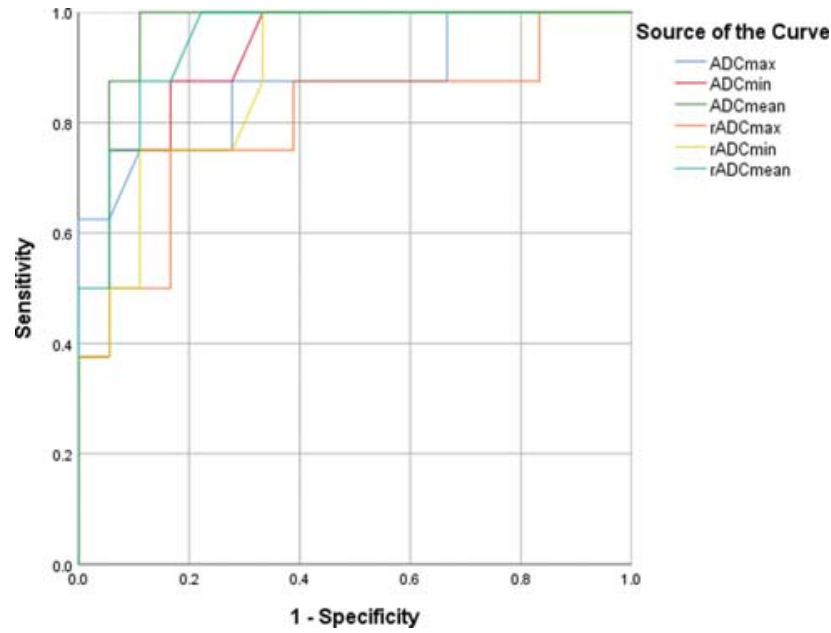

Fig. 3 The receiver operating characteristic curves for the maximum apparent diffusion coefficient (ADC), minimum ADC, mean ADC, the ratios between the tumor and parenchyma values for each $A D C$ were also calculated (rADCmax, rADCmin, and rADCmean values).

flow inside of capillaries and the successful distribution mechanisms through the membrane. Dense cellular tumors have fewer interstitial spaces, resulting in decreased water diffusion and lower ADC values; thus, DWI may be useful when measuring diffusivity level of brain tumor tissues, which is among the most significant variables when evaluating tumor types and grades. ${ }^{7-13}$ In our study, the ADCmax, ADCmin, ADCmean, rADCmax, rADCmin, and rADCmean values for DIPGs were significantly higher than those for fourth ventricular ependymomas $(p<0.05)$. A cutoff ADCmean value of $1.02 \times 10^{-3} \mathrm{~mm}^{2} / \mathrm{s}$ was determined for the differential diagnosis between fourth ventricular ependymomas and DIPGs, yielding the highest sensitivity value of $100 \%$, a specificity of $88.9 \%$, and an AUC of $95.8 \%$.

Numerous researches have proposed the application of multiple ADC values to assist in the identification of ependymomas and DIPGs. However, they did not concentrate on the performance of DWI and the cutoff ADC values in the distinction between fourth ventricular ependymoma and DIPG. ${ }^{9-13,16-19}$

Table 2 ROC analysis of ADC and rADC parameters for the differential diagnosis between fourth ventricular ependymomas and diffuse intrinsic pontine gliomas

\begin{tabular}{|l|l|l|l|l|l|}
\hline & Cutoff point & AUC & Sensitivity & Specificity & $95 \% \mathrm{Cl}$ \\
\hline ADC & & & & & \\
\hline ADCmax $\left(10^{-3} \mathrm{~mm}^{2} / \mathrm{s}\right)$ & 1.24 & 0.872 & 0.750 & 0.889 & $0.704-1.000$ \\
\hline ADCmin $\left(10^{-3} \mathrm{~mm}^{2} / \mathrm{s}\right)$ & 0.76 & 0.920 & 0.875 & 0.833 & $0.815-1.000$ \\
\hline ADCmean $\left(10^{-3} \mathrm{~mm}^{2} / \mathrm{s}\right)$ & 1.02 & 0.958 & 1 & 0.889 & $0.884-1.000$ \\
\hline ADC ratio & & & & & \\
\hline rADCmax & 1.57 & 0.799 & 0.750 & 0.833 & $0.592-1.000$ \\
\hline rADCmin & 1.41 & 0.885 & 1 & 0.667 & $0.757-1.000$ \\
\hline rADCmean & 1.58 & 0.948 & 1 & 0.778 & $0.869-1.000$ \\
\hline
\end{tabular}

Abbreviations: $A D C$, apparent diffusion coefficient; $A U C$, area under the receiver operating characteristic curve; $95 \% \mathrm{Cl}, 95 \%$ confidence interval; $A D C$ ratio, the ratio between the tumor and parenchyma values for each $A D C$ variable; maxADC, maximum $A D C ;$ minADC, minimum ADC; ROC, receiver operating characteristic. 
According to the study reported by Chen et al, which examined nine patients with DIPG, the mean ADC value was $1.14 \pm 0.18 \times 10^{-3} \mathrm{~mm}^{2} / \mathrm{s}^{16}$ More recently, Duc reported that the median diffusivity value of 15 DIPGs was $1.28 \times 10^{-3} \mathrm{~mm}^{2}$ / s. ${ }^{17}$ Additionally, Lober et al reported that the median ADC value for 20 DIPGs was $1.29 \times 10^{-3} \mathrm{~mm}^{2} / \mathrm{s}^{18}$ Therefore, based on three previous studies, the ADC values of DIPG ranged from 1.14 to $1.29 \times 10^{-3} \mathrm{~mm}^{2} / \mathrm{s} .{ }^{16-18}$ In contrast, Jaremko et al ${ }^{19}$ reported that the range of ADC values for 5 ependymomas was 0.65 to $0.99 \times 10^{-3} \mathrm{~mm}^{2} / \mathrm{s}$, and Zitouni et al ${ }^{12}$ suggested that the mean ADC value for 10 ependymomas was $1.03 \pm 0.210^{-3} \mathrm{~mm}^{2} / \mathrm{s}$. Esa et $\mathrm{al}^{10}$ reported a mean $\mathrm{ADC}$ value for 12 ependymomas of $1.04 \pm 0.210^{-3} \mathrm{~mm}^{2} / \mathrm{s}$. In addition, Rumboldt et al observed an ADC value of $1.10 \pm 0.110^{-3} \mathrm{~mm}^{2} / \mathrm{s}$ among five ependymomas. ${ }^{9}$ Based on these previous reports, the ADC values for ependymoma ranged from 0.65 to $1.10 \times 10^{-3} \mathrm{~mm}^{2} / \mathrm{s}^{9,10,12,19}$ In this present study, the ADCmean value for fourth ventricular ependymoma was $0.77 \times 10^{-3} \mathrm{~mm}^{2} / \mathrm{s}$, whereas the median ADCmean value for DIPG was $1.38 \times 10^{-3} \mathrm{~mm}^{2} / \mathrm{s}$. Our findings are fully consistent with those reported by previous studies.

These variations between fourth ventricular ependymoma and DIPG ADC values can be clarified by examining the underlying principle associated with DWI and ADC measurements. DWI measures the Brownian motion of water molecules inside a tissue voxel. Unlike the free flow of water, diffusion inside any given tissue is limited by the boundaries created by cell membranes. A single voxel's overall diffusion role represents the sum of all water diffusion that occurs intracellularly, extracellularly, and in all cellular compartments present in a tissue. The intracellular space comprises the spaces within human cells, including the cytoplasm and the organelles, whereas the extracellular space includes spaces within the intravascular, lymphatic, interstitial, and intracavitary areas. Any increases in cellular mass, irregular compounds, or large particles within certain spaces can reduce the diffusion coefficient. ${ }^{9-20}$ Ependymoma, a mostly solid tumor, is graded as II or III according to the World Health Organization (WHO) system. Some ependymomas are noted to expand cellular density, mitoses, necrosis, and microvascular propagation while keeping the typical pattern of ependymoma. Basically, reductions in the water's free flow, due to limited inter- and intracellular spaces, lead to a cutback of diffusion's signal output. Therefore, the speed of apparent diffusion in a solid, dense tumor like ependymomas, would be small. ${ }^{9,10,12,19,21}$ Inversely, gliomas in the brain stem are less condensed. The intercellular space is usually larger, such that the protons of hydrogen are not greatly restrained. Both of these factors would prompt considerably higher ADC values in DIPG than in ependymoma. ${ }^{16-18}$

Our research is associated with some limitations, including small sample size and the inclusion of only one center. In addition, in this study, researcher only purposed to utilize DWI to differentiate fourth ventricular ependymoma from DIPG; therefore, the knowledge of morphological characteristics and conventional MRI features was insufficient. Furthermore, according to our hospital protocols, the DIPG cases were properly diagnosed and treated based on agreement of neuroradiologists and neurosurgeons, despite the lack of histopathological tests, for tumor type confirmation that was a major drawback of this research. We suggest that further studies should be performed to validate our current results, including the use of broader sample sizes and multicenter participation. Furthermore, future studies with the combination of conventional MRI and DWI are very crucial to enhance the distinct diagnosis between these tumor types.

\section{Conclusion}

The cutoff ADCmean value of $1.02 \times 10^{-3} \mathrm{~mm}^{2} / \mathrm{s}$ was determined to be the most effective parameter for differentiating between fourth ventricular ependymoma and DIPG, with a sensitivity value of $100 \%$, a specificity of $88.9 \%$, and an AUC of 95.8\%. Other variables such as ADCmax, ADCmin, rADCmax, rADCmin, and rADCmean values might also aid radiologists to discriminate between these two tumor types. To support our current results, more studies, including larger sample sizes and the participation of multiple centers, should be implemented.

\section{Highlights}

1. Fourth ventricular ependymoma can trigger a misdiagnosis of DIPG in certain clinical conditions.

2. The median ADCmax, ADCmin, ADCmean, rADCmax, rADCmin, and rADCmean values were significantly lower in fourth ventricular ependymoma than in DIPG.

3. The ADCmean was a critical and effective factor for differentiating between pediatric fourth ventricular ependymoma and DIPG.

Ethical Approval and Declaration of Patient Consent Institutional review board of Children's Hospital 2 approved this prospective study (Ref: 352/NĐ2-CĐT). Written informed consent was obtained from the legal guardian of all participating patients.

\section{Data Availability}

Data sharing is not applicable to this article as no new data were created or analyzed in this study.

Funding

None.

\section{Conflicts of Interest}

None delcared.

Acknowledgment

We would like to express our gratitude to Dr Mai Tan Lien Bang, Dr Dang Do Thanh Can, Dr Huynh Quang Huy, Bilgin Keserci, Dang Thi Bich Ngoc, and Nguyen Chanh Thi for their assistance and technical support in completing this research.

\section{References}

1 Ostrom QT, de Blank PM, Kruchko C, et al. Alex's Lemonade Stand Foundation infant and childhood primary brain and central nervous system tumors diagnosed in the United States in 20072011. Neuro-oncol 2015;16(Suppl 10):x1-x36 
2 Duc NM, Huy HQ Nadarajan C, Keserci B. The role of predictive model based on quantitative basic magnetic resonance imaging in differentiating medulloblastoma from ependymoma. Anticancer Res 2020;40(05):2975-2980

3 Kasliwal MK, Agrawal D. Intrinsic brainstem glioma mimicking an ependymoma. Pediatr Radiol 2009;39(11):1250

4 Thakar S, Mohan D, Furtado SV, Ghosal N, Hegde AS. An anaplastic ependymoma presenting as an intrinsic brainstem glioma. Neurol India 2012;60(01):131-132

5 Jallo GI, Biser-Rohrbaugh A, Freed D. Brainstem gliomas. Childs Nerv Syst 2004;20(03):143-153

6 Tonn JC, Reardon DA, Rutka JT, Westphal M. Oncology of CNS Tumors. 3rd edition Switzerland AG: Springer Nature ; 2019:527-528

7 Koob M, Girard N. Cerebral tumors: specific features in children. Diagn Interv Imaging 2014;95(10):965-983

8 Poretti A, Meoded A, Huisman TA. Neuroimaging of pediatric posterior fossa tumors including review of the literature. J Magn Reson Imaging 2012;35(01):32-47

9 Rumboldt Z, Camacho DL, Lake D, Welsh CT, Castillo M. Apparent diffusion coefficients for differentiation of cerebellar tumors in children. AJNR Am J Neuroradiol 2006;27(06):1362-1369

10 Esa MMM, Mashaly EM, El-Sawaf YF, Dawoud MM. Diagnostic accuracy of apparent diffusion coefficient ratio in distinguishing common pediatric CNS posterior fossa tumors. Egypt J Radiol Nucl Med 2020;51:76

11 Yamasaki F, Kurisu K, Satoh K, et al. Apparent diffusion coefficient of human brain tumors at MR imaging. Radiology 2005;235(03): 985-991

12 Zitouni S, Koc G, Doganay S, et al. Apparent diffusion coefficient in differentiation of pediatric posterior fossa tumors. Jpn J Radiol 2017;35(08):448-453
13 Duc NM, Huy HQ. Magnetic resonance imaging features of common posterior fossa brain tumors in children: a preliminary Vietnamese study. Open Access Maced J Med Sci 2019;7(15): 2413-2418

14 Schumacher M, Schulte-Mönting J, Stoeter P, Warmuth-Metz M, Solymosi L. Magnetic resonance imaging compared with biopsy in the diagnosis of brainstem diseases of childhood: a multicenter review. J Neurosurg 2007;106(02):111-119

15 Albright AL. Diffuse brainstem tumors: when is a biopsy necessary? Pediatr Neurosurg 1996;24(05):252-255

16 Chen HJ, Panigrahy A, Dhall G, Finlay JL, Nelson MD Jr, Blüml S. Apparent diffusion and fractional anisotropy of diffuse intrinsic brain stem gliomas. AJNR Am J Neuroradiol 2010;31(10): 1879-1885

17 Duc NM. The role of diffusion tensor imaging metrics in the discrimination between cerebellar medulloblastoma and brainstem glioma. Pediatr Blood Cancer 2020;67:e28468

18 Lober RM, Cho YJ, Tang Y, et al. Diffusion-weighted MRI derived apparent diffusion coefficient identifies prognostically distinct subgroups of pediatric diffuse intrinsic pontine glioma. J Neurooncol 2014;117(01):175-182

19 Jaremko JL, Jans LB, Coleman LT, Ditchfield MR. Value and limitations of diffusion-weighted imaging in grading and diagnosis of pediatric posterior fossa tumors. AJNR Am J Neuroradiol 2010;31 (09):1613-1616

20 Duc NM, Huy HQ Bang MTL, et al. Clinical applications of diffusion-weighted magnetic resonance imaging. Imaging Med 2018;10:79-84

21 Duc NM. The effect of semi-quantitative T1-perfusion parameters for the differentiation between pediatric medulloblastoma and ependymoma. Egypt J Radiol Nucl Med 2020;51:109 